\title{
FOOTNOTE TO THE TITCHMARSH-LINNIK DIVISOR PROBLEM ${ }^{1}$
}

\section{H. HALBERSTAM}

Let $\tau$ denote the divisor function, and define

$$
T_{a}(x)=\sum_{a<p \leqq x} \tau(p-a) .
$$

By means of his dispersion method, Linnik [1] proved the following

Theorem. As $x \rightarrow \infty, T_{a}(x) \sim E x$, where

$$
E=\prod_{p+a}\left(1+\frac{1}{p(p-1)}\right) \prod_{p / a}\left(1-\frac{1}{p}\right) .
$$

The study of $T_{a}(x)$ was initiated as long ago as 1931 by Titchmarsh [2].

Since the dispersion method is exceedingly complicated, it may be of interest to record that the theorem is a simple consequence of the recent result of Bombieri [3] on the average of the error term in the prime number theorem for arithmetic progressions. For our purpose the following, weaker, form of Bombieri's theorem is sufficient:

(i) There exists a positive number B such that

$$
\sum_{d \leqq x^{1 / 2}(\log x)^{-B}} \max _{1 \leqq h<d ;(h, d)=1}\left|\pi(x ; d, h)-\frac{\operatorname{li} x}{\phi(d)}\right| \ll \frac{x}{\log x}
$$

(the notation « indicates an inequality with an unspecified constant factor).

We shall require also the following well-known results from elementary number theory (see Prachar [4, p. 44, Satz 4.1] and Estermann [5] respectively):

(ii) If $1 \leqq d<x, 0 \leqq h<d,(h, d)=1$, then

$$
\pi(x ; d, h) \ll \frac{x}{\phi(d) \log (x / d)}
$$

uniformly in $x$ and $d$.

$$
\sum_{d \leq x ;(d, a)=1} \frac{1}{\phi(d)}=E \log x+O(1) .
$$

Received by the editors June 23, 1966.

${ }^{1}$ Professor Bombieri has pointed out to me that the same observation has already been recorded by G. Rodriguez, Boll. Un. Mat. Ital. in September 1965. 
Proof of Theorem. Since

$$
\tau(n)=2 \sum_{d \mid n ; d<n^{1 / 2}} 1+\theta(n),
$$

where $\theta(n)=1$ if $n$ is a perfect square and is otherwise 0 , it follows that

$$
\begin{aligned}
T_{a}(x) & =2 \sum_{a<p \leq x} \sum_{d \mid(p-a) ; d<(p-a)^{1 / 2}} 1+O\left(x^{1 / 2}\right) \\
& =2 \sum_{d<(x-a)^{1 / 2}} \sum_{a+d^{2}<p \leq x ; p \equiv a(\bmod d)} 1+O\left(x^{1 / 2}\right) \\
& =2 \sum_{d<x^{1 / 2} ;(d, a)=1}\left\{\pi(x ; d, a)-\pi\left(a+d^{2} ; d, a\right)\right\}+O\left(x^{1 / 2}\right) \\
& =2 \sum_{d<x^{1 / 2} ;(d, a)=1} \pi(x ; d, a)+O(x / \log x)
\end{aligned}
$$

by (ii) (with $x=d^{2}+a$ ). Hence, by (i),

$$
\begin{aligned}
& T_{a}(x)=2 \operatorname{li} x \sum_{d \leqq x^{1 / 2}(\log x)^{-B} ;(d, a)=1} \frac{1}{\phi(d)}+\sum_{x^{1 / 2}(\log x)^{-B}<d<x^{1 / 2} ;(d, a)=1} \pi(x ; d, a) \\
&+O(x / \log x)=2 \operatorname{li} x \sum_{d \sum^{1 / 2} ;(d, a)=1} \frac{1}{\phi(d)} \\
&+ O\left(\frac{x}{\log x}\left\{1+\sum_{x^{1 / 2}(\log x)^{-B}<d<x^{1 / 2}} \frac{1}{\phi(d)}\right\}\right)
\end{aligned}
$$

by (ii); and applying (iii) to both sums on the right we arrive at

$$
T_{a}(x)=E x+O\left(x \cdot \frac{\log \log x}{\log x}\right),
$$

and hence the theorem.

\section{BIBLIOGRAPHY}

1. Ju. V. Linnik, The dispersion method in binary additive problems, (Leningrad 1961), Transl. Math. Monographs, Vol. 4, Amer. Math Soc., Providence, R. I., 1963; Chapter 8.

2. E. C. Titchmarsh, Rend. di Palermo 54 (1931), 414-429.

3. E. Bombieri, Mathematika 12 (1965), 201-225.

4. K. Prachar, Primzahlverteilung, Springer, Berlin, 1957.

5. T. Estermann, J. London Math. Soc. 6 (1931), 250-251.

University OF Michigan and

UNIVERSITY OF NotTINGHAM, ENGLAND 